\title{
Infantile Dilated X-Linked Cardiomyopathy, G4.5 Mutations, Altered Lipids, and Ultrastructural Malformations of Mitochondria in Heart, Liver, and Skeletal Muscle
}

\author{
John J. Bissler, Monica Tsoras, Harald H. H. Göring, Peter Hug, Gail Chuck, \\ Esther Tombragel, Catherine McGraw, James Schlotman, Michael A. Ralston, and \\ George Hug
}

Department of Pediatrics (JJB, MT, GC, ET, CM, JS, GH), University of Cincinnati, Cincinnati, and Children's Medical Center (MAR), Dayton, Ohio; Department of Genetics (HHHG), Southwest Foundation for Biomedical Research, San Antonio, Texas; and Section on Membrane Structure and Function (PH), Laboratory of Experimental and Computational Biology, NCl-FCRDC, Frederick, Maryland

\begin{abstract}
SUMMARY: Mutations in the Xq28 gene G4.5 lead to dilated cardiomyopathy (DCM). Differential splicing of G4.5 results in a family of proteins called "tafazzins" with homology to acyltransferases. These enzymes assemble fatty acids into membrane lipids. We sequenced G4.5 in two kindreds with X-linked DCM and in two unrelated men, one with idiopathic DCM and the other with DCM of arrhythmogenic right ventricular dysplasia. We examined the ultrastructure of heart, liver, and muscle biopsy specimens in these three DCM types; we used gas chromatography to compare fatty acid composition in heart, liver, and muscle autopsy specimens of two patients of kindred 1 with that of controls. In X-linked DCM, G4.5 had a stop codon (E188X), a nonsense mutation, in kindred 1 and an amino acid substitution (G240R), a missense mutation, in kindred 2. In the two men with isolated DCM, G4.5 was not mutated. Ultrastructural mitochondrial malformations were present in the biopsy tissues of the patients with DCM. Cardiac biopsy specimens of both kindreds with X-linked DCM exhibited greatly enlarged mitochondria with large bundles of stacked, compacted, disarrayed cristae that differed from those of the two types of isolated DCM. Autopsy tissue of patients with X-linked DCM had decreased unsaturated and increased saturated fatty acid concentrations. Seven of 13 published G4.5 missense mutations, including the one presented here, occur in acyltransferase motifs. Impaired acyltransferase function could result in increased fatty acid saturation that would decrease membrane fluidity. Mitochondrial membrane proliferation may be an attempt to compensate for impaired function of acyltransferase. Cardiac ultrastructure separates X-linked DCM with G4.5 mutations from the two types of isolated DCM without G4.5 mutations. Electron microscopy of promptly fixed myocardial biopsy specimens has a role in defining the differential diagnosis of DCM. Mutational analysis of the G4.5 gene also serves this purpose. (Lab Invest 2002, 82:335-344).
\end{abstract}

$X$ -linked dilated cardiomyopathy (DCM) has variable presentations. In 1979, Neustein et al reported abnormal mitochondria in cardiac myocytes obtained from an infant boy with X-linked DCM who died of heart failure at age 16 months. Barth et al (1983) presented an X-linked DCM with skeletal myopathy, neutropenia, abnormal mitochondria in heart, muscle, and neutrophils, and respiratory chain abnormalities of mitochondria in muscle and cultured fibroblasts (Barth et al, 1996). Kelley et al (1991) described seven boys with X-linked DCM, growth

Received December 12, 2001.

This work was supported by Grant DK0241801 from the National Institute of Diabetes and Digestive and Kidney Diseases and by Grant MO1 RR08084 from the National Institutes of Health.

Address reprint requests to: Dr. John J. Bissler, Children's Hospital Research Foundation \#5, 3333 Burnet Ave, Cincinnati, OH 45229-3039. E-mail: john.bissler@chmcc.org. Current address for Dr.P. Hug: Master Chemical Company, 501 W. Boundary, Perrysburg, OH 43551-1200. retardation, 3-methylglutaconic aciduria, and neutropenia whose initial presentations varied from DCM to isolated neutropenia. Bolhuis et al (1991) localized a disease locus to distal Xq28, and Bione et al (1996) identified mutations in the G4.5 gene. They found that the gene was alternatively spliced, resulting in multiple proteins they termed "tafazzins." D'Adamo et al (1997) analyzed G4.5 in 11 families with X-linked DCM and suggested that the type of mutation influenced the disease phenotype. Johnston et al (1997) analyzed 19 different X-linked DCM pedigrees and found no relationship between type or location of G4.5 mutation and severity of heart disease, neutropenia, or 3-methylglutaconic aciduria. Neuwald (1997) pointed out that tafazzins have acyltransferase homology and suggested that they may be involved in maintaining mitochondrial morphology and function. Vreken et al (2000) studied cultured fibroblasts from patients and concluded that mitochondrial phospholipids may be affected. 
We describe eight boys in two kindreds with X-linked DCM, mutations in G4.5, malformed mitochondria of heart, liver, and muscle, and unusual fatty acid composition. The mitochondrial malformations in these patients differ from those in two unrelated men with isolated DCM, one with idiopathic DCM (Hug and Schubert, 1970) and the other with DCM of arrhythmogenic right ventricular dysplasia (Blankenship et al, 1993). Neither of these two men had a G4.5 mutation.

\section{Results}

\section{Patients}

DCM was diagnosed in patients based on clinical examination, chest x-ray, electrocardiogram, echocardiography, and inheritance (Fig. 1, A and B). Kindred 1 , Patients IV 1 and 9 had growth retardation ( $<5 \%$ for height); kindred 1, Patient IV 2 and kindred 2, Patients V 1 and 3 did not. Kindred 1, Patients IV 1, 2, and 9 and kindred 2, Patients $V 1$ and 3 could be examined for symptoms of skeletal myopathy. None were noted. Neutropenia was not found in any patients. Four patients were tested for urinary 3-methylglutaconic and 3-methylglutaric acids (Duke
University Medical Center, Pediatric Metabolism Laboratory, Raleigh, North Carolina). These urinary acid excretions were moderately increased in kindred 1, Patients IV 1 and 9, but were not increased in kindred 1, Patient IV 2 and kindred 2, Patient V 1. Electron transport chain complexes I, II, III, and IV in heart autopsy tissue of kindred 1 Patient IV 10 were normal as were complexes II, III, and IV in cultured fibroblasts of kindred 1, Patient IV 9 (Clinical Pharmacology Laboratory, Department of Veterans Affairs Medical Center, Cleveland, Ohio).

Kindred 1, Patient IV 8 appeared healthy until age 16 months, when he died after 1 week of an upper respiratory tract infection without fever or edema. At autopsy he had cardiomyopathy of unknown etiology. His brother IV 10 died of DCM at age 23.5 months. Their cousin IV 1 died at age 4 years after 2 days of a viral gastroenteritis. He had appeared healthy while being treated for DCM. Cousin IV 15 died 2 days after birth with hydrops fetalis. Kindred 1, Patients IV 2 and 9 and kindred 2, Patients $\mathrm{V} 1$ and 3 are being treated for DCM at ages 10, 16, 9, and 5 years. Currently they appear clinically healthy and show no symptoms of cardiac failure.

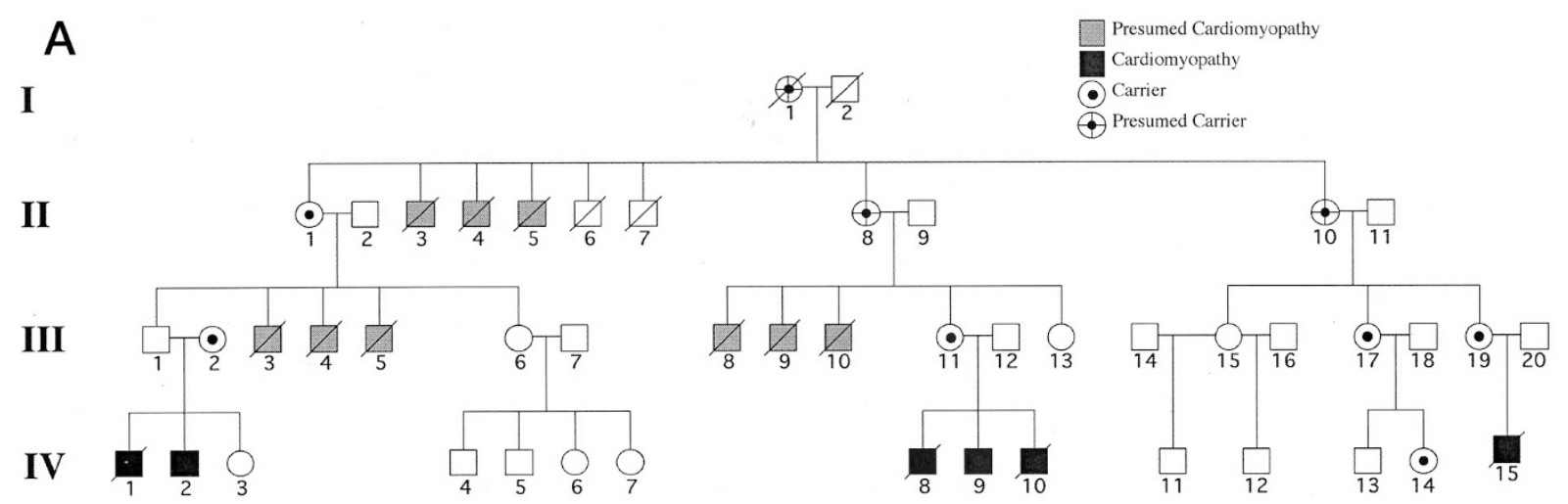

B

I

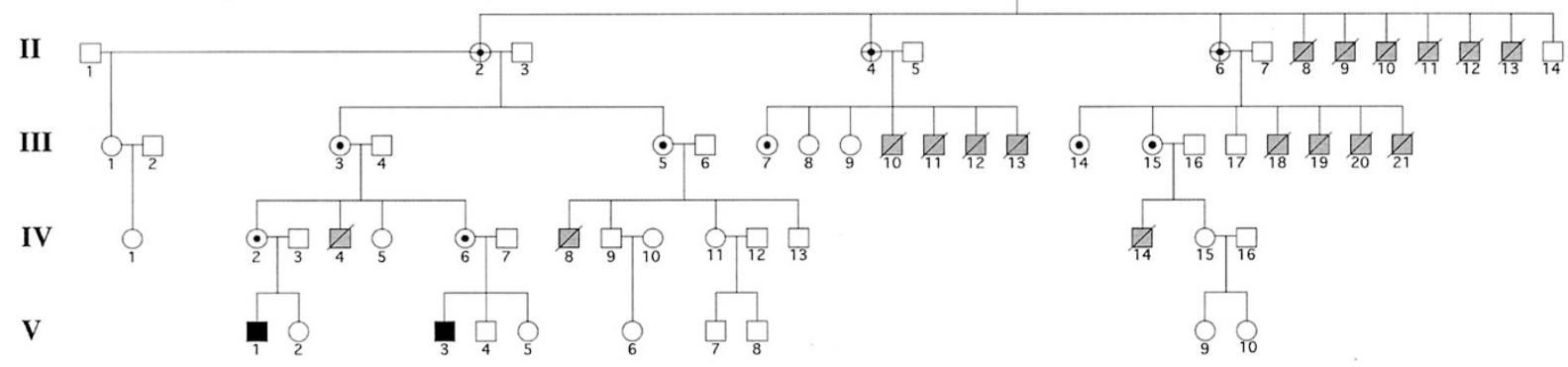

Figure 1.

Eight patients with $\mathrm{X}$-linked dilated cardiomyopathy in two pedigrees. A denotes kindred 1 with the nonsense mutation, and B denotes kindred 2 with the missense mutation in G4.5. 


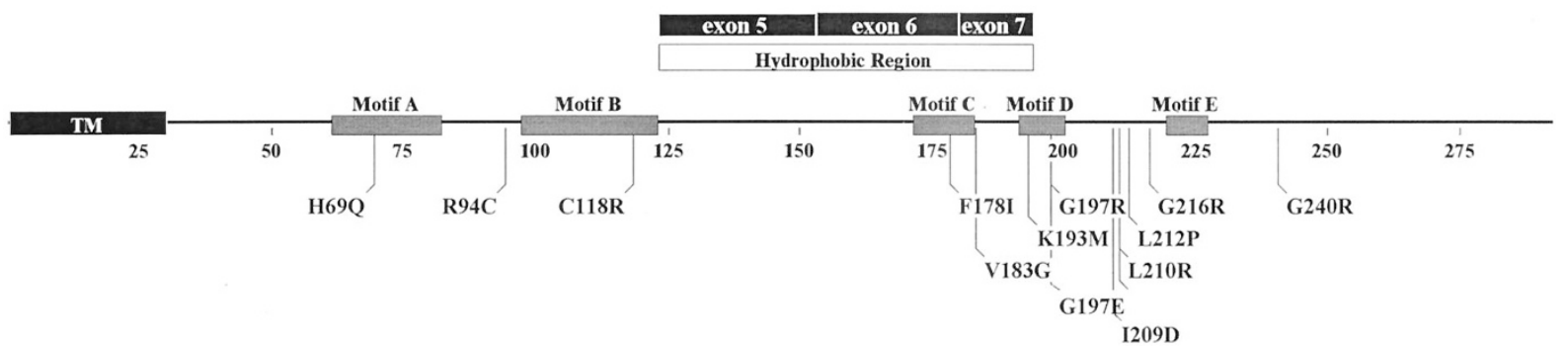

Figure 2.

Full-length tafazzin protein. Regions that are removed alternatively by differential splicing (exon 5,6 , or 7 ) are marked as black boxes. Putative acyltransferase motifs identified by Neuwald (1997) are marked as gray boxes. Missense mutations identified in the literature are labeled by lines extending downward. TM = the putative transmembrane region of the protein.

\section{Mutations in Gene G4.5}

We tested whether the cardiomyopathy in kindred 1 cosegregated with the chromosomal region implicated in Barth syndrome, Xq28, by penetrancemodel-based linkage analysis. Assuming an X-linked recessive disease with full penetrance, and allowing for mutation to occur at the trait locus, a maximum lod score of 3.35 at a recombination fraction of 0 was obtained, providing significant evidence for linkage between the disease locus and Xq28 (Morton, 1955). This indicates that the cardiomyopathy segregating in this pedigree is associated with mutations in the tafazzin gene implicated in Barth syndrome.

When absence of mutation at the trait locus was assumed, the maximum lod score was only 0.02 , obtained at recombination fraction 0.32 . The probable explanation for the difference in the linkage signal is that Patient I 1 carries a novel mutation in the tafazzin gene. It is possible to pinpoint the likely origin of the mutation in this pedigree, because Patient I 1 received marker allele 2 from her unaffected father, which is the marker allele that cosegregates with the disease in the lower generations of the pedigree. (Note that the pedigree drawing in Fig. $1 \mathrm{~A}$ shows only part of the pedigree used for linkage analysis. Neither the parents of Patient I 1 nor observed marker genotypes are indicated.) If no mutations are allowed for in the analysis, obligatory recombination events are inferred, thus deflating the lod score dramatically. Although it is not unexpected from population genetics theory to find evidence for new mutations for $\mathrm{X}$-linked lethal diseases, this pedigree serves as a good example of how linkage may be easily overlooked if the possibility of mutation is not taken into account.

We sequenced the tafazzin gene cDNA and portions of the genomic DNA. In the first kindred, we found a transversion G850T that resulted in a 50\% truncation in the predicted protein size (E188X), and we attribute the disease phenotype to this nonsense mutation because it always cosegregated with the disease phenotype. This mutation always cosegregated with a downstream A866T. Both mutations were found only in patients or carriers of kindred 1 . The second kindred exhibited a single transition G1006A. This missense mutation resulted in the amino acid substitution G240R. No unaffected fam- ily members revealed this mutation, and G240 appeared to be conserved even in Drosophila melanogaster (AE003821), Caenorhabditis elegans (T28003), and Arabidopsis thaliana (AC005679) homologues.

Figure 2 is a diagram of the full-length tafazzin protein. The regions of the alternatively spliced protein without exons 5,6 , or 7 are marked at the top of the illustration. These alternative splices modulate a relatively hydrophobic region of the protein. Acyltransferase motifs identified by $\mathrm{Neu}-$ wald (1997) are marked as gray boxes and comprise a quarter of the full-length protein. Of the 13 pub-

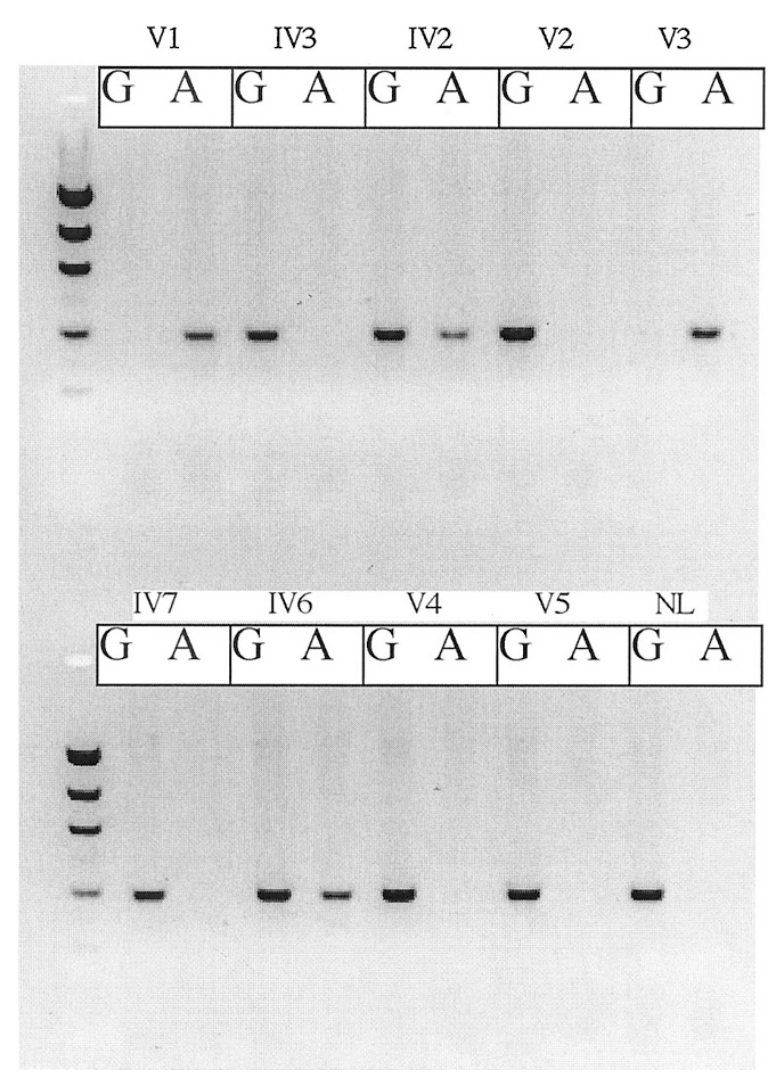

Figure 3.

Mismatch annealing mutation analysis from kindred 2. The complementary oligonucleotide (GGCCAAGAATCCAGAAGGCAGC) was used in conjunction with the oligonucleotide (CACAGAAAATCACTGTGCTCG) for the wild-type allele and (CACAGAAAATCACTGTGCTGATCA) for the mutant allele. Because these last two oligonucleotides differ at their $3^{\prime}$ nucleotide, the mutant and wild-type allele can be identified by the production of a PCR product. 


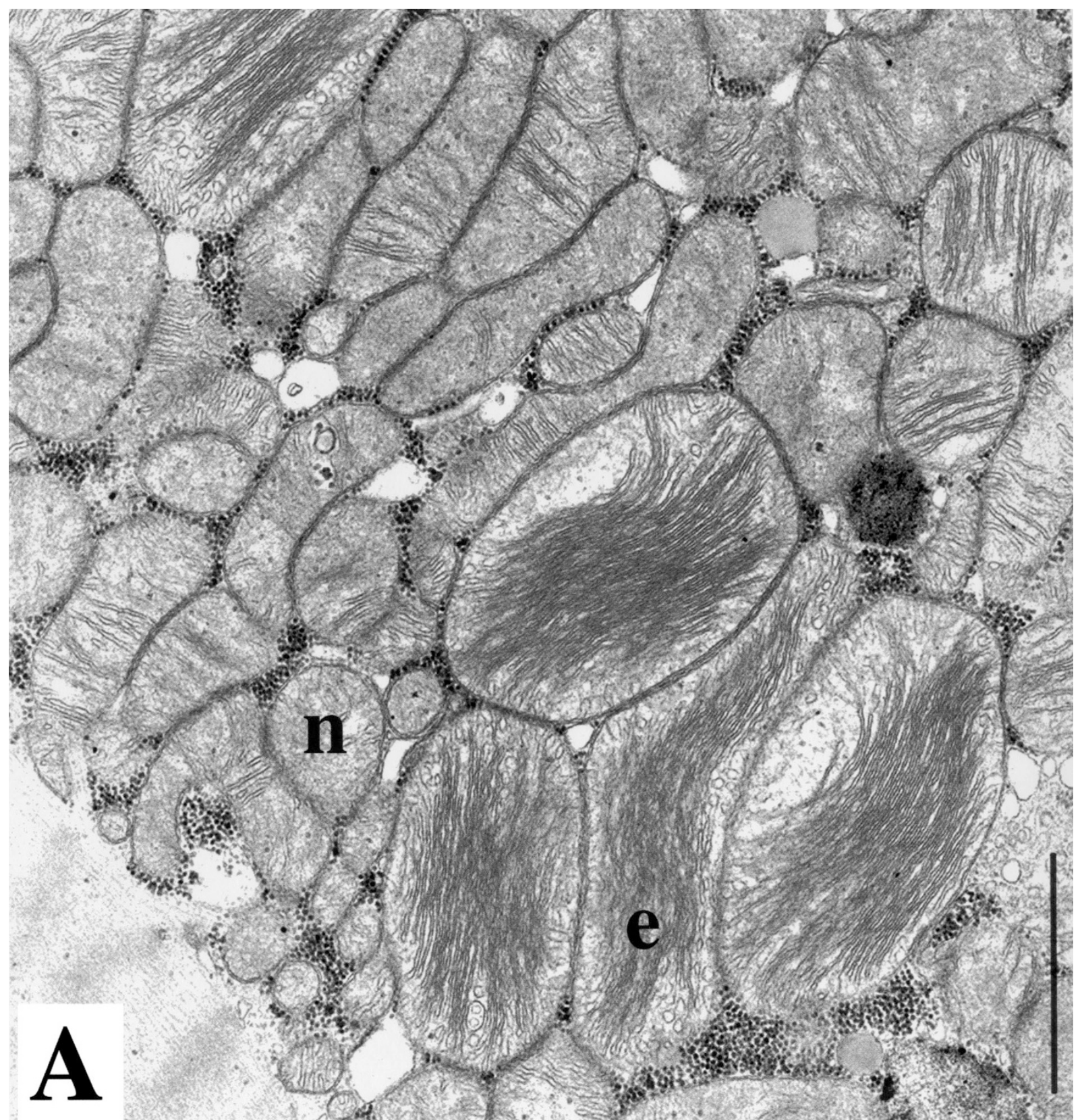

Figure 4.

A, Heart, kindred 2, Patient V 1, bar: $1 \mu \mathrm{m}$. An aggregate of mitochondria is shown. Many mitochondria are normal (n) in size and structure; others are enlarged (e). They contain closely stacked cristae. Intracristal space and matrix space between cristae are narrow and vary in width. Black particles between mitochondria are normal $\beta$-glycogen particles in the sarcoplasm.

lished missense mutations, 7 occur in these motifs. Mutations V183G and G197E have been found repeatedly (Bleyl et al, 1997; Cantlay et al, 1999; D'Adamo et al, 1997; Ichida et al, 2001; Johnston et al, 1997; Neuwald, 1997). The significant clustering of over one-half the reported disease-causing mutations in putative acyltransferase motifs $(p<0.022$, $\chi^{2}$ test) may reveal regions of the tafazzin protein critical for its function. Mutations were analyzed in family members using a mismatch amplification mutation analysis technique. An example for kindred 2 is shown in Figure 3 and can be correlated with Figure 1B.

\section{Histologic Examination}

\section{Light Microscopy}

Liver and muscle did not show diagnostic abnormalities. Cardiac autopsy specimens of kindred 1, Patients IV 1,8 , and 10 showed myocyte hypertrophy, myofiber disarray, focal interstitial fibrosis, and mild endocardial thickening.

\section{Electron Microscopy}

Figure 4, A to E, is representative of the findings in the biopsied tissue of the current patients. Figure $4 \mathrm{~F}$ is 


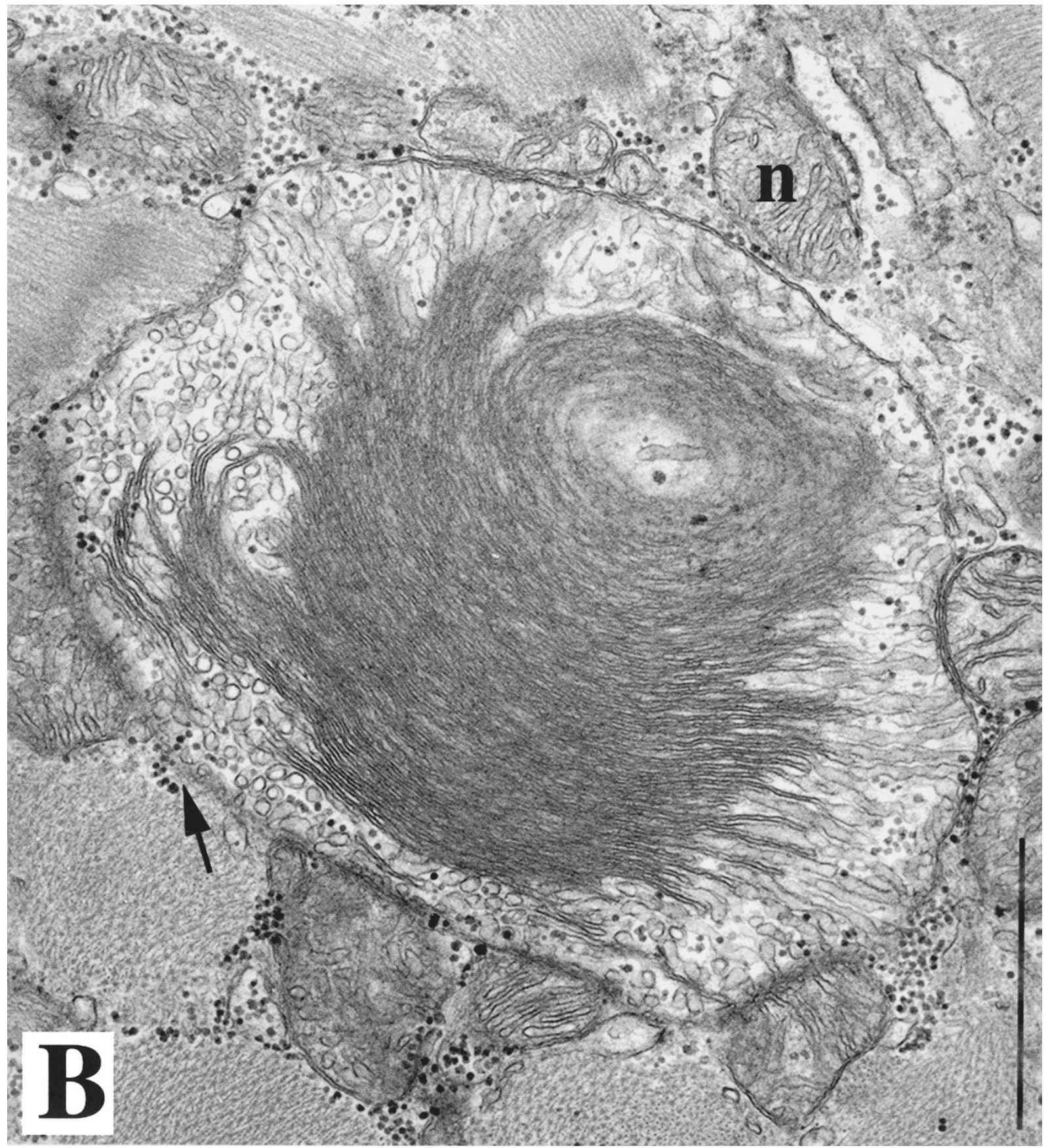

Figure 4.

B, Heart, kindred 1, Patient IV 10, bar: $1 \mu \mathrm{m}$. Several normal mitochondria ( $\mathrm{n}$ ) are adjacent to a greatly enlarged mitochondrion with tightly stacked cristae. These are in disarray. The intracristal space and matrix space between cristae are narrow or absent. Cristae extend into electron-dense material. In gaps of the outer and inner membranes of the enlarged mitochondrion, one sees $\beta$-glycogen particles that extend from sarcoplasm to matrix (arrow).

representative of heart findings of the two men with isolated DCM and normal G4.5 gene. The findings in these two men have been described previously in more detail (Blankenship et al, 1993; Hug and Schubert, 1970).

Heart, current patients. Mitochondria were plentiful and frequently present in large aggregates between myofibrils (Fig. 4, A to C). The majority of mitochondria were normal in size and structure, and contained normal cristae. Many mitochondria had bundles of packed cristae. Some bundles consisted of numerous cristae, others of just a few. A minority of mitochondria were large or very large. Their cristae were closely packed in straight or circular patterns. These cristae appeared disarrayed, disorderly, wavy, and contorted (Fig. 4A).

The largest cardiomyocytic mitochondria (Fig. 4, B and C) had tubular and tightly packed lamellar cristae. Intracristal space and matrix space between adjacent lamellar cristae were narrow and sometimes not detectable. The width of both these spaces varied from crista to crista and along the course of the same two 


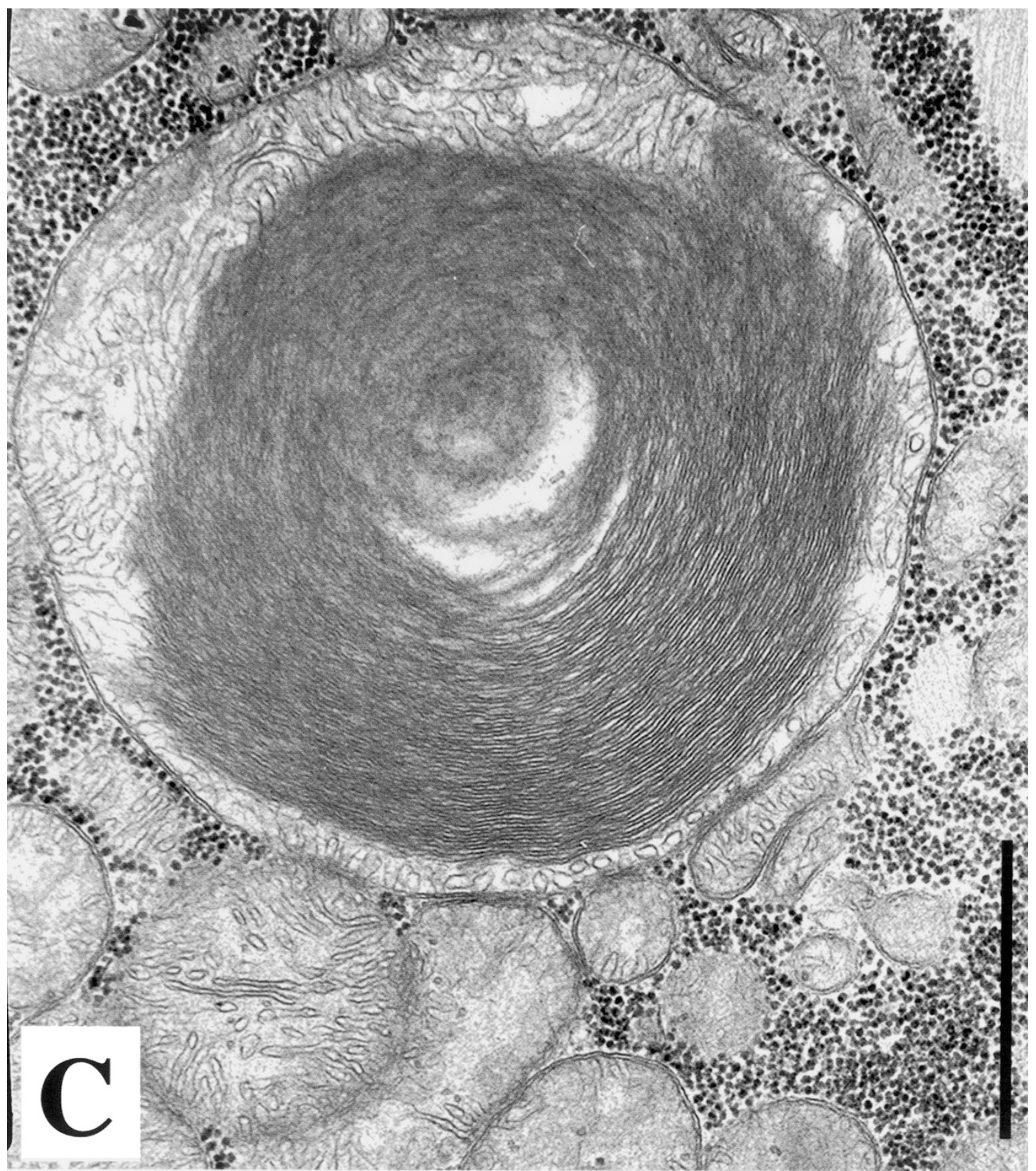

Figure 4.

C, Heart, kindred 2, Patient V 1, bar: $1 \mu \mathrm{m}$. This large mitochondrion is similar to that in B except one sees no gaps in the outer or inner membrane and no glycogen particles in the matrix.

adjacent cristae. Some cristae extended into finely lamellar, feathery or fluffy, electron-dense material. Some of the largest mitochondria had gaps in the inner and outer membranes with $\beta$-glycogen particles extending from cytoplasm to matrix space.

Liver, current patients. Mitochondria were numerous and generally not enlarged (Fig. 4D). Some had bundles of cristae in straight, circular, or angular patterns.

Skeletal muscle, current patients. Mitochondria were numerous, small, and had dense granules in- creased in number and size (Fig. 4E). Some mitochondria contained a few bundled cristae in straight or circular patterns.

Heart, man with idiopathic DCM heart. Mitochondria were plentiful (Fig. 4F). Some were enlarged. The majority had abundant, closely stacked cristae. Even in the largest mitochondria these cristae were orderly and not in disarray. They were positioned parallel to each other in straight or circular patterns. The width of the intracristal space was constant, as was that of the 

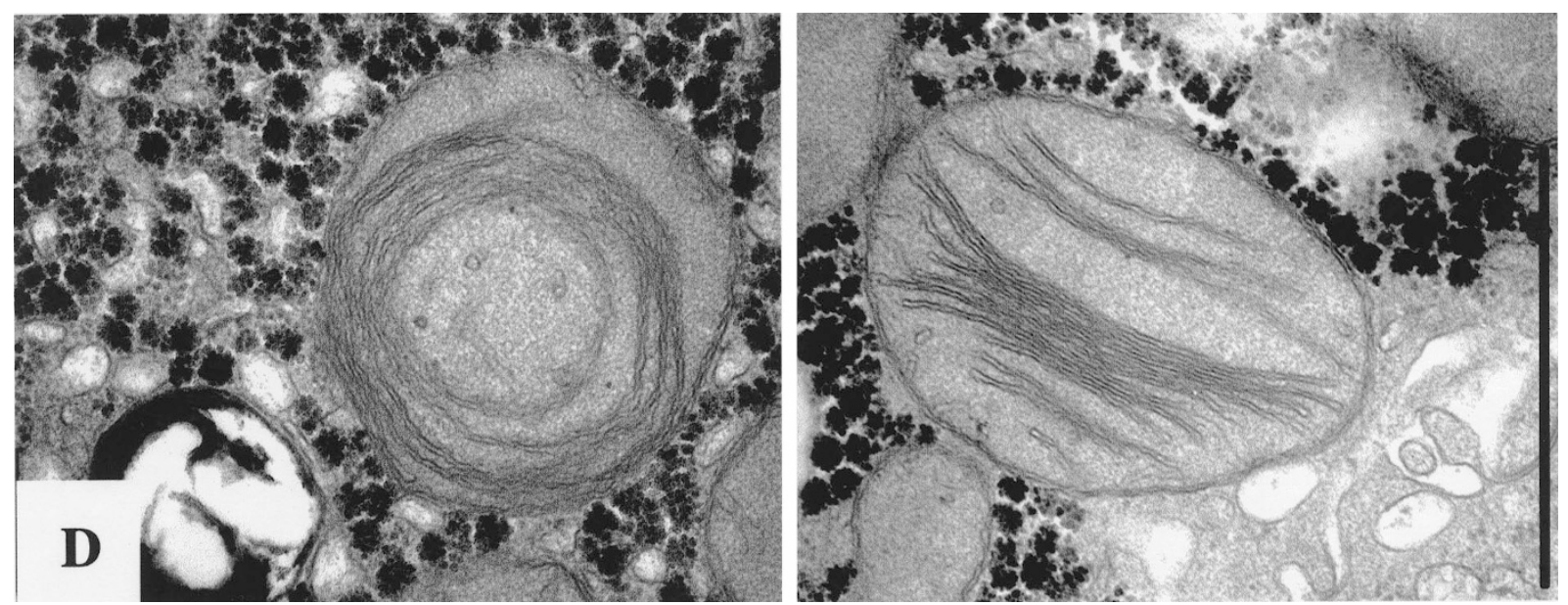

Figure 4.

D, Liver, kindred 1, Patient IV 9, bar: $1 \mu \mathrm{m}$. Many hepatocytic mitochondria are normal (not shown). These two mitochondria are typical of the changes seen in the patients. Both mitochondria are normal in size but have straight and circular bundles of closely aligned cristae. The black particles are normal $\alpha$-glycogen particles.

matrix space between adjacent lamellar cristae. The matrix was often floccular. Cristae were absent in floccular areas. The findings in Figure $4 \mathrm{~F}$ are not distinguishable from those of the man with arrhythmogenic right ventricular dysplasia. We did not encounter any large mitochondria similar to those of the current patients in these two men with isolated DCM. The mitochondria of the current patients were separable from those of the two men. Neutrophils were examined in one patient from each kindred and appeared normal.

\section{Fatty Acid Analysis}

In Table 1, fatty acid concentrations in autopsy tissues of heart, liver, and muscle of kindred 1, Patients IV 1 and 10 are listed that differ from those of controls $(p<$ 0.05). Fatty acids of chain length $C 12$ to $C 22$ exhibited concentrations that were different in patients and controls. In the patients, the concentrations of four unsaturated fatty acids were increased and those of 17 were decreased; whereas, the concentrations of 13 saturated fatty acids were increased and none were decreased. In brief, patient tissues contained decreased unsaturated and increased saturated fatty acids.

\section{Discussion}

Kindred 1 had a nonsense mutation (E188X) and kindred 2 a missense mutation (G240R) in G4.5. The two different mutations produced indistinguishable mitochondrial malformations. The eight patients were being treated similarly for X-linked cardiomyopathy. Four patients who had the nonsense mutation died. None of our patients had skeletal myopathy or neutropenia. Some patients of both kindred had growth retardation or increased 3-methylglutaconic aciduria; others did not. Respiratory chain abnormalities, reported in other patients with X-linked DCM (Barth et al, 1983, 1996), were not found in our patients. Relating genotype to phenotype has been difficult previously
(Cantlay et al, 1999; D'Adamo et al, 1997; Johnston et al, 1997) and remains difficult today.

Mitochondrial malformations were present in all biopsy specimens. The malformations were pronounced in heart but less marked in liver and muscle. These lesions were similar for the same organ in both kindred. We conclude that DCM with G4.5 mutations exhibits mitochondrial malformations that can be used in defining the differential diagnosis of DCM.

This conclusion is strengthened by the differently appearing stacked cristae of cardiomyocytic mitochondria found in the two unrelated males with isolated DCM. One was a 6-month-old, adopted boy (Hug and Schubert, 1970) who 30 years later still had idiopathic DCM with malformed mitochondria. The other man died at the age of 59 years of arrhythmogenic right ventricular dysplasia (Blankenship et al, 1993). Both patients' cardiac mitochondrial malformations appeared similar and are inseparable from each other (Blankenship et al, 1993; Hug and Schubert, 1970; and Fig. 4F), but they are substantially different and separable from those of the current patients (Fig. 4, A to C). We analyzed G4.5 in both men with isolated DCM and found no mutations.

Ichida et al (2001) and Bleyl et al (1997) reported G4.5 mutations in some of their patients with left ventricular noncompaction (LVNC). One could hypothesize that LVNC patients with G4.5 mutations would have the mitochondrial changes of our current patients, whereas LVNC patients lacking G4.5 mutations would not. In any event, the evaluation of endomyocardial biopsy specimens of DCM should include electron microscopy. Useful ultrastructural information can be obtained when the specimens are placed in the fixative less than 2 minutes after the biopsy.

Neuwald et al (1997) identified six regions of the tafazzin protein with acyltransferase homology. $\mathrm{He}$ proposed that tafazzin proteins might be involved in phospholipid biosynthesis and remodeling and that this process, if defective because of mutations in 

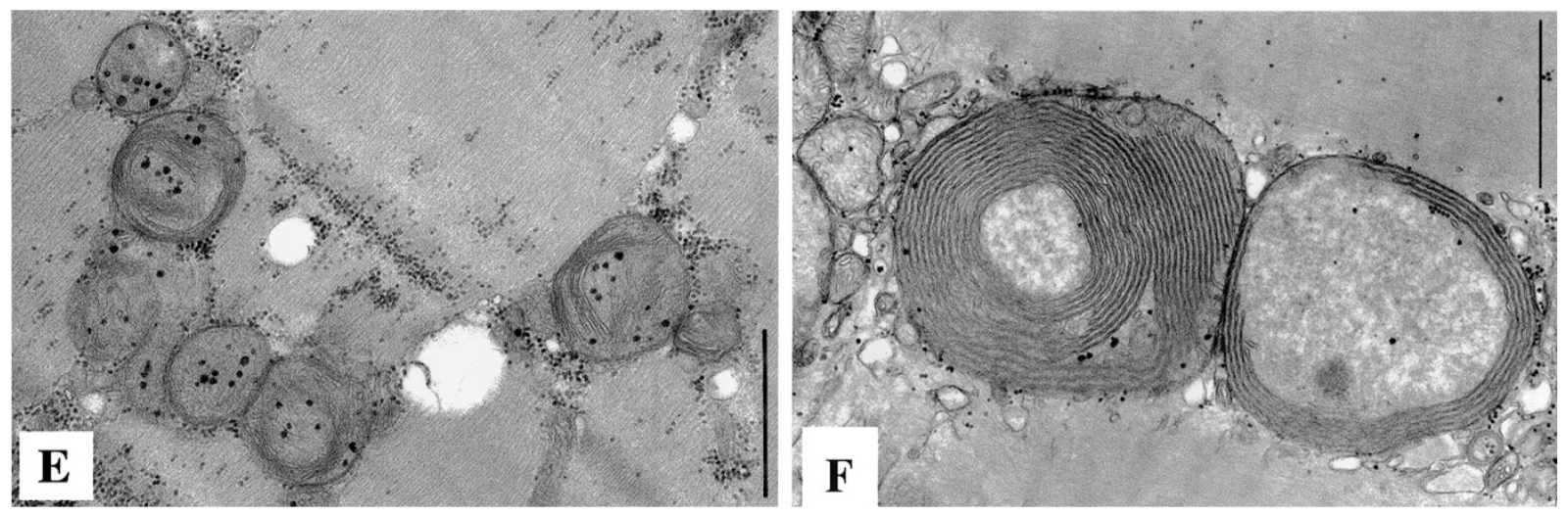

Figure 4.

E, Skeletal muscle, kindred 1, Patient IV 10, bar: $1 \mu \mathrm{m}$. The seven identifiable mitochondria are small; three have bundles of circular, closely aligned cristae and five have between 5 and 10 prominent dense granules. F, Heart biopsy specimen from the man with idiopathic dilated cardiomyopathy (DCM) (Hug and Schubert, 1970). Findings do not differ and are not separable from those in the heart of the man with arrhythmogenic right ventricular dysplasia (Blankenship et al, 1993). Bar: $1 \mu \mathrm{m}$. Circular and straight bundles of aligned, parallel cristae maintain the width of intracristal space and of matrix space in between adjacent cristae. These changes affect the majority of mitochondria and are different and separable from those of the current patients.

G4.5, might lead to malformed mitochondrial membranes (Neuwald, 1997). The unusual fatty acid composition in autopsy tissues of kindred 1, Patients IV 1 and 10 may be consistent with this proposal. Patient tissue had more saturated and less unsaturated fatty acids than did controls (Table 1). In skeletal and cardiac muscle, a large fraction of esterified fatty acids is derived from phospholipid. The acyl moieties of phospholipid molecules determine many of the physical properties of the membrane. The degree of unsaturation is important in determining membrane fluidity, a measure of the ease of movement of molecules within the membrane bilayer. Membrane fluidity at a given temperature is inversely proportional to the fraction of acyl chain moieties comprised of saturated fatty acids.

Cellular membrane fluidities are maintained within a narrow range by acyltransferase-mediated remodeling of phospholipid within the membrane. If the tafazzin gene product is an acyltransferase present within the mitochondrial membrane, these membranes in affected patients may have reduced fluidity, possibly reducing the normal function of integral membrane proteins. The activity of proteins such as lecithin:cholesterol acyltransferase is affected by changes in membrane fluidity (Parks et al, 2000).

Proliferation of inner mitochondrial membrane may be in response to reduced mitochondrial unit efficiency. The proliferation may boost mitochondrial function to near normal. The proliferation could result in enlarged mitochondria, in overabundant, dissolving cristae, and in mitochondrial membrane disintegration. These are the observed mitochondrial changes in the cardiomyocytes of our patients. With mitochondrial function only marginally sufficient, the clinical outcome may depend in part on environmental factors such as dietary fatty acids, carnitine (Barth et al, 1983; Ostman-Smith et al, 1994), or pantothenic acid (Ostman-Smith et al, 1994).

\section{Patients and Methods}

\section{Patients}

In the current generation of kindred 1 (Fig. 1A), six boys had DCM (Patients IV 1, 2, 8, 9, 10, and 15); and of kindred 2 (Fig. 1B), two boys had DCM (Patients V 1 and 3). Tissue specimens of heart (by endomyocardial biopsy), muscle, and liver (by open or needle biopsy) were obtained for diagnostic studies from kindred 1, Patients IV 1, 9, and 10, and kindred 2, Patient V 1. Autopsy tissue (frozen or fixed in formalin or glutaraldehyde) was available from kindred $1, \mathrm{~Pa}-$ tients IV 1, 8, 10, and 15. DNA isolation from leukocytes and/or tissue specimens was performed in the eight patients and in potential carrier females.

\section{Genetic Analysis}

Linkage Analysis. Because of similarities of our patients' disease with Barth syndrome (Barth et al, 1983), we performed penetrance-model-based linkage analysis with DXS52, a marker $1 \mathrm{MB}$ from G4.5, using the LINKAGE software package (Lathrop et al, 1984). The disease was assumed to be an X-linked fully penetrant recessive disease without phenocopies. The frequency of the disease-causing allele was set to 0.0001 . A mutation rate of $1 / 3$ of this allele frequency was assumed (Haldane, 1935). Individuals in whom X-linked cardiomyopathy was suspected but not confirmed as the cause of death were coded as having unknown disease status. Published allele frequency estimates were used for genotype marker DXS52 (Richards et al, 1991).

Molecular Analysis. RNA was isolated from normal tissue and from patient heart and liver using the Qiagen RNeasy total RNA kit (Qiagen, Valencia, California). Reverse transcription was carried out using the Perkin Elmer Thermostable rTth Reverse Transcriptase RNA PCR kit and primers as described by Bione et al (1996). 


\begin{tabular}{|c|c|c|c|c|c|c|c|c|}
\hline \multirow[b]{2}{*}{ Lipid name } & \multirow{2}{*}{$\begin{array}{l}\text { Chain } \\
\text { length }\end{array}$} & \multirow[b]{2}{*}{$\Delta$} & \multicolumn{3}{|c|}{ Esterified } & \multicolumn{3}{|c|}{ Free } \\
\hline & & & Patient & Control & $p$ Value & Patient & Control & $p$ Value \\
\hline \multicolumn{9}{|l|}{ Left ventricle } \\
\hline Stearic & 18 & 0 & $20.42 \pm 4.80$ & $8.52 \pm 7.39$ & 0.037 & $17.98 \pm 7.74$ & $7.09 \pm 4.46$ & 0.004 \\
\hline Eicosatrienoic & 20 & 3 & 0.00 & $0.25 \pm 0.55$ & 0.039 & 0.00 & $0.13 \pm 0.24$ & 0.018 \\
\hline Eicosapentaenoic & 20 & 5 & $0.76 \pm 0.08$ & $0.01 \pm 0.04$ & 0.001 & $0.84 \pm 0.56$ & $0.023 \pm 0.08$ & 0.289 \\
\hline Docosapentaenoic & 22 & 5 & 0.00 & $0.28 \pm 0.32$ & 0.001 & 0.00 & $0.24 \pm 0.28$ & 0.001 \\
\hline Docosahexaenoic & 22 & 6 & $1.36 \pm 0.28$ & $1.74 \pm 1.09$ & 0.632 & $0.95 \pm 0.01$ & $1.47 \pm 0.63$ & 0.001 \\
\hline \multicolumn{9}{|l|}{ Right ventricle } \\
\hline Lauric & 12 & 0 & $4.45 \pm 1.04$ & $0.48 \pm 1.09$ & 0.001 & $2.14 \pm 0.44$ & $0.70 \pm 1.03$ & 0.064 \\
\hline Myristic & 14 & 0 & $5.30 \pm 6.77$ & $2.45 \pm 5.94$ & 0.52 & $7.40 \pm 1.171$ & $1.81 \pm 3.11$ & 0.021 \\
\hline Palmitic & 16 & 0 & $40.42 \pm 8.30$ & $25.83 \pm 49.49$ & 0.687 & $46.19 \pm 1.61$ & $16.62 \pm 19.05$ & 0.021 \\
\hline Stearic & 18 & 0 & $24.72 \pm 1.28$ & $8.52 \pm 7.39$ & 0.006 & $25.27 \pm 2.57$ & $7.09 \pm 4.46$ & 0.001 \\
\hline Eicosatrienoic & 20 & 3 & 0.00 & $0.25 \pm 0.55$ & 0.039 & 0.00 & $0.13 \pm 0.24$ & 0.018 \\
\hline Docosapentaenoic & 22 & 5 & 0.00 & $0.28 \pm 0.32$ & 0.001 & 0.00 & $0.24 \pm 0.28$ & 0.001 \\
\hline Docosahexaenoic & 22 & 6 & $1.85 \pm 0.97$ & $1.74 \pm 1.09$ & 0.900 & $0.50 \pm 0.68$ & $1.47 \pm 0.63$ & 0.044 \\
\hline \multicolumn{9}{|l|}{ Liver } \\
\hline Myristic & 14 & 0 & $14.01 \pm 7.52$ & $3.29 \pm 7.25$ & 0.024 & $13.78 \pm 4.56$ & $3.17 \pm 5.45$ & 0.004 \\
\hline Palmitic & 16 & 0 & $136.72 \pm 17.63$ & $38.49 \pm 70.62$ & 0.026 & $135.51 \pm 27.73$ & $30.98 \pm 47.89$ & 0.001 \\
\hline Stearic & 18 & 0 & $45.06 \pm 15.15$ & $12.19 \pm 13.52$ & 0.001 & $42.42 \pm 2.1$ & $9.94 \pm 10.44$ & 0.001 \\
\hline Docosapentaenoic & 22 & 5 & 0.00 & $0.17 \pm 0.28$ & 0.008 & 0.00 & $0.14 \pm 0.26$ & 0.014 \\
\hline Oleic & 18 & 1 & $149.58 \pm 58.11$ & $49.58 \pm 110.16$ & 0.139 & $127.65 \pm 47.35$ & $35.48 \pm 64.38$ & 0.025 \\
\hline Arachidonic & 20 & 4 & $20.23 \pm 5.96$ & $10.72 \pm 11.33$ & 0.17 & $15.46 \pm 5.22$ & $7.00 \pm 6.52$ & 0.041 \\
\hline \multicolumn{9}{|l|}{ Muscle } \\
\hline Linoleic & 18 & 2 & $16.6 \pm 2.97$ & $42.82 \pm 29.16$ & 0.003 & $10.7 \pm 8.10$ & $29.65 \pm 22.97$ & 0.184 \\
\hline Linolenic & 18 & 3 & 0.00 & $1.4 \pm 2.14$ & 0.022 & $0.17 \pm 0.29$ & $1.56 \pm 2.06$ & 0.021 \\
\hline Eicosadienoic & 20 & 2 & $0.68 \pm 0.63$ & $0.09 \pm 0.35$ & 0.027 & $0.31 \pm 0.54$ & $0.035 \pm 0.14$ & 0.471 \\
\hline Eicosatrienoic & 20 & 3 & 0.0 & $0.43 \pm 0.52$ & 0.006 & 0.00 & $0.15 \pm 0.25$ & 0.031 \\
\hline
\end{tabular}

In patient autopsy tissues, saturated fatty acids are increased and unsaturated fatty acids are decreased. List, by name and number of unsaturated bonds $(\Delta)$ in means $\pm 1 \mathrm{SD} \mu \mathrm{g} / \mathrm{mg}$ tissue protein, of the free or esterified tissue fatty acids (FA) of chain length C12 to C22 that differ between patients and controls $(p<0.05)$. Dark boxes list the $10 \mathrm{FA}$ that differ in either the free or esterified form but not in both. Four unsaturated FA are increased and 17 are decreased; 13 saturated FA are increased and none are decreased.

The sequencing of cDNA from one patient in kindred 1 yielded a sequence that lacked exon 7 (nucleotides 655-954; GenBank accession number X92764) in contrast to cDNAs from normal controls. Therefore, sequencing of genomic DNA including splice junctions of exon 7 was performed on patient DNA. Sequencing cDNA from a patient in kindred 2 was successful in identifying a missense mutation. Nonaffected family members were analyzed for this mutation, and a Blast search analysis (NCBI) was undertaken to determine the conservation of the amino acid involved in the missense mutation. Previously described primers were used in the terminator cycle sequencing as described in the Perkin-Elmer protocol (no. 402116).

After sequencing identified the mutation sites in the two kindreds, we used a mismatch annealing mutation amplification technique to confirm the mutation identification and to screen other family members (Cha et al, 1992). Briefly, three PCR primers were used. One of them was a complementary oligonucleotide, and the two others were designed so that one contained the wild-type base and the other contained the mutant base in the $3^{\prime}$ location. These two primers were used in separate PCR reactions with the complementary oligonucleotide. Individuals with only the wild type, or normal, allele yielded an amplification product only with the wild-type oligonucleotide. Male patients with a mutant allele yielded a product only with the mutant oligonucleotide, whereas carriers with both alleles would yield a product with both the mutant and the wild-type oligonucleotide.

\section{Histologic Examination}

Specimens of autopsy and biopsy of heart, liver, and muscle were embedded for light and electron microscopic evaluation. For the latter we placed biopsy specimens in glutaraldehyde within 1 to 2 minutes after removal from the body. This is critical because delayed fixation or the use of autopsy tissue specimens can result in significant artifacts. Cardiac findings in the current patients were compared with those in two unrelated men who had isolated DCM without G4.5 mutations (Blankenship et al, 1993; Hug and Schubert, 1970).

\section{Fatty Acid Analysis}

Free and esterified fatty acids were determined by gas chromatography and expressed in micrograms per milligram of tissue protein. The Hewlett Packard GC system (HP 5890A) had a flame ionization detector 
and an HP Ultra2 (cross-linked 5\% Ph Me Silicone $25 \mathrm{~m} \times 0.32 \mathrm{~mm} \times 0.25 \mu \mathrm{m}$ film) capillary column capable of separating the methyl ester derivatives of $6: 0,8: 0,10: 0,12: 0,14: 0,16: 0,16: 1 \mathrm{c}, 16: 1 \mathrm{t}, 18: 0$, 18:1c, 18:1t, 18:2, 18:3, 20:0, 20:1, 20:2, 20:3, 20:4, $20: 5,22: 0,22: 1,22: 4,22: 5,22: 6,24: 0,24: 1,26: 0$ (Ackman et al, 1972; Russell et al, 1974). The assay was calibrated with a methyl ester mixture (GLC-30, Supelco); methyl heptadecanoate was used as an internal standard. Results in autopsy tissues of kindred 1, Patients IV 1 and 10 were compared by independent group $t$ test with those of patients without defective lipid metabolism.

\section{References}

Ackman RG, Hooper SN, and Hansen RP (1972). Some monomethyl-branched fatty acids from ruminant fats: opentubular GLC separations and indications of substitution on even-numbered carbon. Lipids 7:683-691.

Barth PG, Scholte HR, Berden JA, Van der Klei-Van Moorsel JM, Luyt-Houwen IE, Van't Veer-Korthof ET, Van der Harten JJ, and Sobotka-Plojhar MA (1983). An X-linked mitochondrial disease affecting cardiac muscle, skeletal muscle and neutrophil leucocytes. J Neurol Sci 62:327-355.

Barth PG, Van den Bogert C, Bolhuis PA, Scholte HR, van Gennip AH, Schutgens RB, and Ketel AG (1996). X-linked cardioskeletal myopathy and neutropenia (Barth syndrome): respiratory-chain abnormalities in cultured fibroblasts. J Inherit Metab Dis 19:157-160.

Bione S, D'Adamo P, Maestrini E, Gedeon AK, Bolhuis PA, and Toniolo D (1996). A novel X-linked gene, G4.5. is responsible for Barth syndrome. Nat Genet 12:385-389.

Blankenship DC, Hug G, Balko G, van der Bel-Kann J, Coith RL Jr, and Engel PJ (1993). Hemodynamic and myocyte mitochondrial ultrastructural abnormalities in arrhythmogenic right ventricular dysplasia. Am Heart J 126:989-995.

Bleyl SB, Mumford BR, Thompson V, Carey JC, Pysher TJ, Chin TK, and Ward K (1997). Neonatal, lethal noncompaction of the left ventricular myocardium is allelic with Barth syndrome. Am J Hum Genet 61:868-872.

Bolhuis PA, Hensels GW, Hulsebos TJ, Baas F, and Barth PG (1991). Mapping of the locus for X-linked cardioskeletal myopathy with neutropenia and abnormal mitochondria (Barth syndrome) to Xq28. Am J Hum Genet 48:481-485.

Cantlay AM, Shokrollahi K, Allen JT, Lunt PW, Newbury-Ecob RA, and Steward CG (1999). Genetic analysis of the G4.5 gene in families with suspected Barth syndrome. J Pediatr 135:311-315.

Cha RS, Zarbl H, Keohavong P, and Thilly WG (1992). Mismatch amplification mutation assay (MAMA): application to the $\mathrm{c}-\mathrm{H}$ - ras gene. PCR Methods Appl 2:14-20.

D'Adamo P, Fassone L, Gedeon A, Janssen EA, Bione S, Bolhuis PA, Barth PG, Wilson M, Haan E, Orstavik KH, Patton MA, Green AJ, Zammarchi E, Donati MA, and Toniolo D (1997). The X-linked gene G4.5 is responsible for different infantile dilated cardiomyopathies. Am J Hum Genet 61:862867.
Haldane $\mathrm{J}$ (1935). The rate of spontaneous mutations of a human gene. J Genetics 31:317-326.

Hug G and WK Schubert (1970). Idiopathic cardiomyopathy. Mitochondrial and cytoplasmic alterations in heart and liver. Lab Invest 22:541-552.

Ichida F, Tsubata S, Bowles KR, Haneda N, Uese K, Miyawaki T, Dreyer WJ, Messina J, Li H, Bowles NE, and Towbin JA (2001). Novel Gene Mutations in Patients With Left Ventricular Noncompaction or Barth Syndrome. Circulation 103:1256-1263.

Johnston J, Kelley RI, Feigenbaum A, Cox GF, lyer GS, Funanage VL, and Proujansky R (1997). Mutation characterization and genotype-phenotype correlation in Barth syndrome. Am J Hum Genet 61:1053-1058.

Kelley RI, Cheatham JP, Clark BJ, Nigro MA, Powell BR, Sherwood GW, Sladky JT, and Swisher WP (1991). X-linked dilated cardiomyopathy with neutropenia, growth retardation, and 3-methylglutaconic aciduria. J Pediatr 119:738747.

Lathrop GM, Lalouel JM, Julier C, and Ott J (1984). Strategies for multilocus linkage analysis in humans. Proc Natl Acad Sci USA 81:3443-3446.

Morton NE (1955). Sequential tests for the detection of linkage. Am J Human Genet 7:277-318.

Neustein HB, Lurie PR, Dahms B, and Takahashi M (1979). An X-linked recessive cardiomyopathy with abnormal mitochondria. Pediatrics 64:24-29.

Neuwald AF (1997). Barth syndrome may be due to an acyltransferase deficiency [letter]. Curr Biol 7:R465-R466.

Ostman-Smith I, Brown G, Johnson A, and Land JM (1994). Dilated cardiomyopathy due to type II X-linked 3-methylglutaconic aciduria: successful treatment with pantothenic acid. Br Heart J 72:349-353.

Parks JS, Huggins KW, Gebre AK, and Burleson ER (2000). Phosphatidylcholine fluidity and structure affect lecithin: cholesterol acyltransferase activity. J Lipid Res 41:546-553.

Richards RI, Shen Y, Holman K, Kozman H, Hyland VJ, Mulley JC, and Sutherland GR (1991). Fragile X syndrome: diagnosis using highly polymorphic microsatellite markers. Am J Hum Genet 48:1051-1057.

Russell PT, Miller WJ, and McLain CR (1974). Palmitic acid content of amniotic fluid lecithin as an index to fetal lung maturity. Clin Chem 20:1431-1434.

Vreken P, Valianpour F, Nijtmans LG, Grivell LA, Plecko B, Wanders RJ, and Barth PG (2000). Defective remodeling of cardiolipin and phosphatidylglycerol in Barth syndrome." Biochem Biophys Res Commun 279:378-382. 